\title{
V. Ueber die N-Alkylketoxime;
}

von Johannes Scheiber und Paul Brandt.

Nachdem sich, wie in der vorhergehenden Mittheilung auseinandergesetzt ist, aus dem kryoscopischen Verhalten einer Auflösung von

bzw.

$$
\text { Keton + Benzylhydroxylamin }
$$

$$
\text { Keton + Benzylhydroxylamin + Base }
$$

in einem geeigneten Lösungsmittel (Benzol) die Fühigkeit der meisten untersuchten Ketone zur Bildung von $\mathrm{N}$-Benzylketoximhydraten ergeben hatte, wurde unter Zngrundelegung der gleichen Nethode auch versucht, einiges Ziffernmiässiges über das Reactionsvermögen der einzelnen Ketone zu erfahren, und zwar sowohl bei Abwesenheit als auch bei Gegenwart basischer substanzen.

A. Ueber das Verhalten von Ketonen gegenüber ß-Benzylhydroxylamin bei Abresenheit von Basen.

Was zunächst den ersten Theil der Aufgabe anging, so war einfach derart zu verfahren, dass man äquimolekulare Mengen der verschiedenen Ketone mit jedesmal der gleichen Menge $\beta$-Benzylhydroxylamin zur Reaction brachte.

Als Lösungsmittel wurde aus den schon früher angegebenen Gründen wiederum Benzol gewählt. Besondere Sorgfalt wurde dabei auf die Trocknung des käuflichen Präparates (Merck) gelegt. Zur Anwendung kamen für alle Versuche durchgängig je $15 \mathrm{~g}$. 
Da es natürlich kaum möglich war, genau molekulare Mengen der Reactionscomponenten abzuwägen und verlustlos in die Gefrierapparate $\mathrm{zu}$ bringen, wurden benzolische Lösungen von ihnen hergestellt, deren Gehalt an Substanz und Lösungsmittel genau bekannt war.

Die zur Yerwendung gelangenden Ketone und zwar: Aceton, Nethyläthylketon, Methylbutylketon, Methylhexylketon, Diäthylketon, Methylbenzylketon und Acetophenon wurden von Kahlbaum bezogen; theils genügte ihre Reinheit, theils wurden sie auch einer nochmaligen Trocknung und Rectification unterworfen.

Ausdrücklich erwähnt sei auch, daß das benöthigte $\beta$-Benzylhydroxylamin für jede Versuchsreihe frisch dargestellt und mit besonderer Sorgfalt gereinigt wurde.

Die einzelnen Bestimmungen wurden in folgender Weise ausgeführt:

Vom Benzylhydroxylamin wurde durchgängig 1 Millimol $(0,1230 \mathrm{~g})$ fïr jeden einzelnen Versuch verwendet. Die Menge der Ketone betrug 1, 2 und 5 Millimol.

Auf Grund des bekannten Gehaltes der Lösungen der einzelnen Componenten wurden die oben angegebenen Mengen auf ccm der betreffenden Lösungen umgerechnet und diese dann aus engen genauen Büretten direct in die Gefrierrohre hinein abgemessen. Eine weitere kurze Rechnung ergab, wieviel Lösungsmittel in den in das Gefrierrohr gebrachten Lösungsmengen enthalten war. Die Differenz zwischen dieser Benzolmenge und $15 \mathrm{~g}$ $(17,26 \mathrm{ccm})$ Benzol wurde zuletzt noch an reinem Lösungsmittel zugegeben.

Die Zeit des Zusammengebens der einzelnen Lösungen wurde notirt; die Zeiten jeder Ablesung des Gefrierpunktes wurden ebenfalls vermerkt und in eine Tabelle eingetragen. Die Versuchsdauer wurde dabei auf ca. $3 \times 24$ Stunden ausgedehnt.

Erwähnt sei ferner, dass der Nullpunkt am Thermometer, d. h. der Punkt, an dem sich das Quecksilber 
beim Gefrierenlassen des reinen Lösungsmittels einstellte nnd von dem an die Depressionen gerechnet wurden, vor jeder Versuchsreihe nen bestimmt wurde. Die Aufbewahrung der gefüllten Gefrierrohre geschah nicht im Kühlbad, sondern bei Zimmertemperatur an einem vor jeglicher Frschiitterung gesicherten Orte.

Un die Genauigkeit der Messungen möglichst sicher zu stellen, wurde die Ausfiuhrung einiger Vor- bzw. Controllversuche für nothwendig erachtet.

So wurden zunächst nochmals Bestimmungen mit den Ketonen allein durchgeführt, damit die den jeweilig angewendeten Ketonmengen möglichst entsprechenden Concentrationen kryoscopisch untersucht waren. Die derart ermittelten ('onstantenwerthe $\left.\left(C=\frac{\mathrm{M} \cdot \mathrm{L} \cdot \Delta}{\log \mathrm{g}}\right)^{2}\right)$ fanden alsdaun Verwendung bei der Berechnung der zu erwartenden Gesamtdepressionen. Auf eine Mittheilung des Zahlenmaterials kann an dieser Stelle indess woh] verzichtet werden Sodann wurden Controllbestimmungen mit Benzylhydroxylamin allein angestellt, um dessen Verhalten bei der Concentration von 1 Millimol in $15 \mathrm{~g}$ Benzol zu beobachten. Denn es war trotz sorgfältigster Reinigung bei der Zersetzlichkeit des Products von vornherein nicht ausgeschlossen, dass bei längerer Reactionsdauer Aenderung der Depression eintrat. Das Benzylhydroxylamin wurde zu diesen Untersuchungen den gleichen Lösungen entnommen, welche für die Ausfïhrung der Versuche mit den Ketonen selbst benutzt wurden. Die jeweils erhaltenen Zahlenserien, deren Mitheilung hier ohne Interesse ist, liessen indess die gehegten Befürchtungen grundlos erscheinen.

Ueber das unter Beobachtung aller dieser Vorsichtsmassregeln erhaltene Zahlenmaterial bei den Combinationen

Keton + Benzylhydroxylamin im Mol.-Verhältnis

$$
1: 1 \text { bzw. } 5: 1
$$

orientirt die folgende Tabelle III:

2) $\mathrm{M}=$ Molekulargewicht. $\mathrm{L}=$ Menge des Lösungsmittels in $\mathbf{g}$.

$\triangle=$ Depression. $\quad \mathrm{g}=$ Substanzmenge. $\mathrm{C}=$ Constante. 
Scheiber und Brandt, $N$-Alliylletoxime.

Tabelle III:

Einwirkung von Ketonen auf Benzylhydroxylamin im Mol.-Terh. $1: 1$ und $5: 1$.

\begin{tabular}{|c|c|c|c|c|c|c|}
\hline Keton & $\begin{array}{l}\text { Mol.- } \\
\text { Verh. }\end{array}$ & $\triangle_{\text {berechn }}$ & $\triangle$ beob. & $\Delta_{\text {bet. }} \triangle_{\text {beob. }}$ & $\left.\triangle_{0}-\triangle_{t}{ }^{3}\right)$ & $\begin{array}{c}\text { Versuchs- } \\
\text { dauer }\end{array}$ \\
\hline \multirow{2}{*}{ Aceton } & $1: 1$ & $0,558^{\circ}$ & $\begin{array}{l}0,561^{\circ} \\
0,538^{\circ} \\
0,475^{\circ} \\
0,438^{\circ}\end{array}$ & $\begin{array}{l}+0,003^{\circ} \\
-0,020^{\circ} \\
-0,083^{\circ} \\
-0,120^{\circ}\end{array}$ & $\begin{array}{c}- \\
-0,023^{\circ} \\
-0,086^{\circ} \\
-0,123^{\circ}\end{array}$ & $\begin{array}{r}0 \text { h. } 10^{\prime} \\
23 \text { h. } 15^{\prime} \\
45 \text { h. } 40^{\prime} \\
69 \text { h. } 15^{\prime}\end{array}$ \\
\hline & $5: 1$ & $1,828^{\circ}$ & $\begin{array}{l}1,770^{\circ} \\
1,623^{\circ} \\
1,608^{\circ} \\
1,607^{\circ}\end{array}$ & $\begin{array}{r}-0,058^{\circ} \\
-0,205^{\circ} \\
-0,220^{\circ} \\
-0,221^{\circ}\end{array}$ & $\begin{array}{l}- \\
-- \\
-\end{array}$ & $\begin{array}{c}4 \text { h. } \\
23 \text { h. } 30^{\prime} \\
45 \text { h. } 50^{\prime} \\
70 \text { h. } 35^{\prime}\end{array}$ \\
\hline \multirow{2}{*}{$\begin{array}{c}\text { Methyl- } \\
\text { athyl- } \\
\text { keton }\end{array}$} & $1: 1$ & $0,552^{\circ}$ & $\begin{array}{l}0,571^{\circ} \\
0,575^{\circ} \\
0,572^{\circ} \\
0,562^{\circ}\end{array}$ & $\begin{array}{l}+0,019^{\circ} \\
+0,029^{\circ} \\
+0,020^{\circ} \\
+0,010^{\circ}\end{array}$ & $\begin{array}{c}- \\
+0,004^{\circ} \\
+0,001^{\circ} \\
-0,009^{\circ}\end{array}$ & $\begin{array}{l}0 \text { h. } 10^{\prime} \\
24 \text { h. } \\
47 \text { h. } 20^{\prime} \\
69 \text { h. } 45^{\prime}\end{array}$ \\
\hline & $5: 1$ & $1.855^{\circ}$ & $\begin{array}{l}1,851^{\circ} \\
1,779^{\circ} \\
1,712^{\circ} \\
1,667^{\circ}\end{array}$ & $\begin{array}{r}-0,004^{\circ} \\
-0,076^{\circ} \\
-0,143^{\circ} \\
-0,188^{\circ}\end{array}$ & $\begin{array}{c}- \\
-0,072^{\circ} \\
-0,139^{\circ} \\
-0,184^{\circ}\end{array}$ & $\begin{array}{c}0 \text { h. } 10^{\prime} \\
24 \text { h. } 5^{\prime} \\
47 \text { h. } 30^{\prime} \\
69 \text { h. } 55^{\prime}\end{array}$ \\
\hline \multirow{2}{*}{$\begin{array}{l}\text { Methyl } \\
\text { butyl- } \\
\text { keton }\end{array}$} & $1: 1$ & $0,556^{\circ}$ & $\begin{array}{l}0,566^{\circ} \\
0,568^{\circ} \\
0,550^{\circ} \\
0,537^{\circ}\end{array}$ & $\begin{array}{c}- \\
-0,006^{\circ} \\
-0,019^{\circ}\end{array}$ & $\begin{array}{l}- \\
+0,002^{\circ} \\
-0,016^{\circ} \\
-0,029^{\circ}\end{array}$ & $\begin{array}{r}0 \text { h. } 10^{\prime} \\
16 \text { h. } 50^{\prime} \\
46 \text { h. } 25^{\prime} \\
63 \text { h. } 50^{\prime}\end{array}$ \\
\hline & $\begin{array}{l}! \\
! \\
! \\
!\end{array}$ & $1,540^{\circ}$ & $\begin{array}{l}1,580^{\circ} \\
1,498^{\circ} \\
1,425^{0} \\
1,406^{\circ}\end{array}$ & $\begin{array}{c}- \\
-0,042^{\circ} \\
-0,115^{\circ} \\
-0,134^{\circ}\end{array}$ & $\begin{array}{c}- \\
-0,082^{\circ} \\
-0,155^{\circ} \\
-0,174^{\circ}\end{array}$ & $\begin{array}{l}0 \text { b. } 10^{\prime} \\
17 \text { h. - } \\
46 \text { h. } 35^{\prime} \\
64 \text { h. }-\end{array}$ \\
\hline $\begin{array}{c}\text { Methyl- } \\
\text { hexyl- } \\
\text { keton }\end{array}$ & $1: 1$ & $0,572^{\circ}$ & $\begin{array}{l}0,658^{\circ} \\
0,668^{\circ} \\
0,672^{\circ} \\
0,644^{\circ}\end{array}$ & $\begin{array}{l}- \\
- \\
-\end{array}$ & $\begin{array}{r}- \\
+0,010^{\circ} \\
+0,014^{\circ} \\
-0,014^{\circ}\end{array}$ & $\begin{array}{c}0 \text { h. } 10^{\prime} \\
15 \text { h. } 35^{\prime} \\
39 \text { h. } 25^{\prime} \\
63 \text { h. } 35^{\prime}\end{array}$ \\
\hline
\end{tabular}

3) Für obige Tabelle III bedeuten: $\triangle_{0}=$ erste der beobachteten Ablesungen; $\Delta_{t}=$ spätere Ablesungen. 
Tabelle IIT:

\begin{tabular}{|c|c|c|c|c|c|c|}
\hline Keton & $\begin{array}{l}\text { Mol.- } \\
\text { Verh. }\end{array}$ & $\triangle_{\text {berechn }}$ & $\triangle_{\text {beob. }}$ & $\triangle_{\text {her. }} \triangle_{\text {beol. }}$ & $\left.\triangle_{0}^{-} \triangle_{t}{ }^{3}\right)$ & $\begin{array}{l}\text { Versuchs- } \\
\text { dauer }\end{array}$ \\
\hline $\begin{array}{l}\text { Methyl- } \\
\text { hexyl- } \\
\text { keton }\end{array}$ & $5: 1$ & $1,898^{\circ}$ & $\begin{array}{l}1,902^{\circ} \\
1,872^{\circ} \\
1,790^{\circ} \\
1,756^{\circ}\end{array}$ & $\begin{array}{c}- \\
-0,026^{\circ} \\
-0,108^{\circ} \\
-0,142^{\circ}\end{array}$ & $\begin{array}{c}- \\
-0,030^{\circ} \\
-0,112^{\circ} \\
-0,146^{\circ}\end{array}$ & $\begin{array}{r}0 \text { h. } 30^{\prime} \\
15 \text { h. } 55^{\prime} \\
39 \text { h. } 50^{\prime} \\
63 \text { h. } 20^{\prime}\end{array}$ \\
\hline \multirow{2}{*}{$\begin{array}{c}\text { Diäthyl- } \\
\text { keton }\end{array}$} & $1: 1$ & $0,561^{\circ}$ & $\begin{array}{l}0,646^{\circ} \\
0,654^{\circ} \\
0,656^{\circ} \\
0,649^{\circ}\end{array}$ & $\begin{array}{l}- \\
- \\
-\end{array}$ & $\begin{array}{r}-\overline{0,008^{\circ}} \\
+0,010^{\circ} \\
+0,003^{\circ}\end{array}$ & $\begin{array}{r}0 \text { h. } 10^{\prime} \\
15 \text { h. } 35^{\prime} \\
39 \text { h. } 30^{\prime} \\
64 \text { h. } 20^{\prime}\end{array}$ \\
\hline & $5: 1$ & $1,88^{\circ}$ & $\begin{array}{l}1,882^{\circ} \\
1,872^{\circ} \\
1,852^{\circ} \\
1,819^{\circ}\end{array}$ & $\begin{array}{l}-0,006^{\circ} \\
-0,016^{\circ} \\
-0,036^{\circ} \\
-0,069^{\circ}\end{array}$ & $\begin{array}{c}- \\
-0,010^{\circ} \\
-0,030^{\circ} \\
-0,063^{\circ}\end{array}$ & $\begin{array}{c}0 \text { h. } 25^{\prime} \\
16 \text { h. } \\
39 \text { h. } 25^{\prime} \\
64 \text { h. } 45^{\prime}\end{array}$ \\
\hline \multirow{2}{*}{$\begin{array}{c}\text { Methyl- } \\
\text { benzyl- } \\
\text { keton }\end{array}$} & $1: 1$ & $0,564^{0}$ & $\begin{array}{l}0,576^{\circ} \\
0,456^{\circ} \\
0,452^{\circ} \\
0,418^{\circ}\end{array}$ & $\begin{array}{r}- \\
-0,108^{\circ} \\
-0,112^{\circ} \\
-0,116^{\circ}\end{array}$ & $\begin{array}{c}- \\
-0,120^{\circ} \\
-0,124^{\circ} \\
-0,128^{\circ}\end{array}$ & $\left(\begin{array}{c}0 \text { h. } 15^{\prime} \\
\left.16 \text { h. } 25^{\prime *}\right) \\
\left.41 \text { h. } 25^{\prime *}\right) \\
\left.66 \text { h. } 25^{\prime *}\right)\end{array}\right.$ \\
\hline & $5: 1$ & $1,735^{0}$ & $\begin{array}{l}1,501^{\circ} \\
1,495^{\circ} \\
1,515^{\circ} \\
1,524^{0}\end{array}$ & $\begin{array}{c}-0,234^{\circ} \\
-0,240^{\circ} \\
-\end{array}$ & $\begin{array}{l}- \\
- \\
-\end{array}$ & $\left(\begin{array}{cc}0 & \text { h. } 25^{\prime * *} \\
17 & \left.\text { h. } 30^{\prime *}\right) \\
41 & \left.\text { h. } 45^{\prime *}\right) \\
66 & \left.\text { h. } 35^{\prime *}\right)\end{array}\right.$ \\
\hline \multirow{2}{*}{$\begin{array}{l}\text { Aceto- } \\
\text { phenon }\end{array}$} & $1: 1$ & $0,575^{\circ}$ & $\begin{array}{l}0,682^{\circ} \\
0,708^{\circ} \\
0,729^{\circ} \\
0,742^{\circ}\end{array}$ & $\begin{array}{c}- \\
- \\
+0,1670\end{array}$ & $\begin{array}{c}-\overline{0,026^{\circ}} \\
+0,047^{0} \\
+0,060^{\circ}\end{array}$ & $\begin{array}{c}0 \text { h. } 15^{\prime} \\
15 \text { h. } 30^{\prime} \\
41 \text { h. } 25^{\prime} \\
66 \text { h. } 35^{\prime}\end{array}$ \\
\hline & $5: 1$ & $1,895^{\circ}$ & $\begin{array}{l}1,890^{\circ} \\
1,905^{\circ} \\
1,915^{\circ} \\
1,916^{\circ}\end{array}$ & $\begin{array}{c}-0,005 \\
- \\
+0,0210\end{array}$ & $\begin{array}{c}- \\
+0,015^{\circ} \\
+0,025^{\circ} \\
+0,026^{\circ}\end{array}$ & $\begin{array}{r}0 \text { h. } 40^{\prime} \\
16 \text { h. } 45^{\prime} \\
41 \text { h. } 45^{\prime} \\
66 \text { h. } 50^{\prime}\end{array}$ \\
\hline
\end{tabular}

*) Gelbfärbang. **) Trübung.

3) Siehe die Fußnote auf der vorhergehenden Seite. 
Eine anschauliche Darstellung der erzielten Versuchsergebnisse ist durch Construction der Depressionsabnahme-Zeit-Kurven möglich. Trägt man nämlich die Werthe für $\triangle$ ber. $-\triangle$ beob. als Ordinaten, die nach Stunden gerechneten Zeiten hingegen als Abscissen in ein Coordinatensystem ein, so gelangt man für die Molekularverhältnisse 1:1 zur Figur 1, für die Mrolekularverhältnisse 5:1 hingegen zur Figur 2,4)

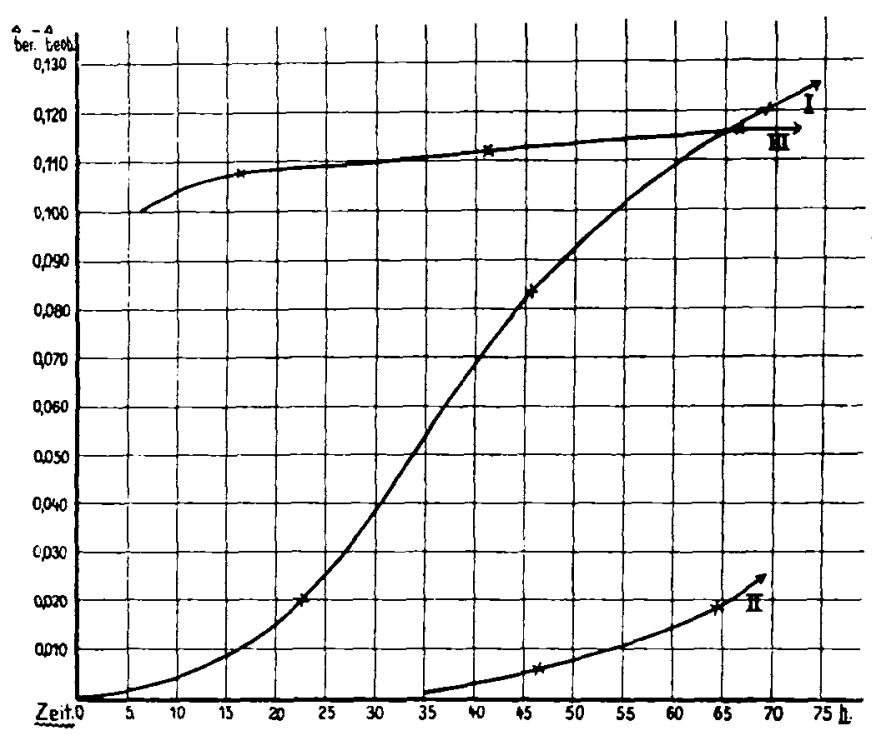

Figar 1.
1. Benzylhydroxylamin + Aceton;
Mol.-Verh.: $1: 1$
II. ,
+ Methylbutylketon; "
$1: 1$
III.
+ Methylbenzylketon; "
$1: 1$.

4) Die resultirenden Kurren bleiben fast unverändert, wenn man als Ordinaten die Werthe für $\triangle_{0}-\triangle_{t}$ benutat. 


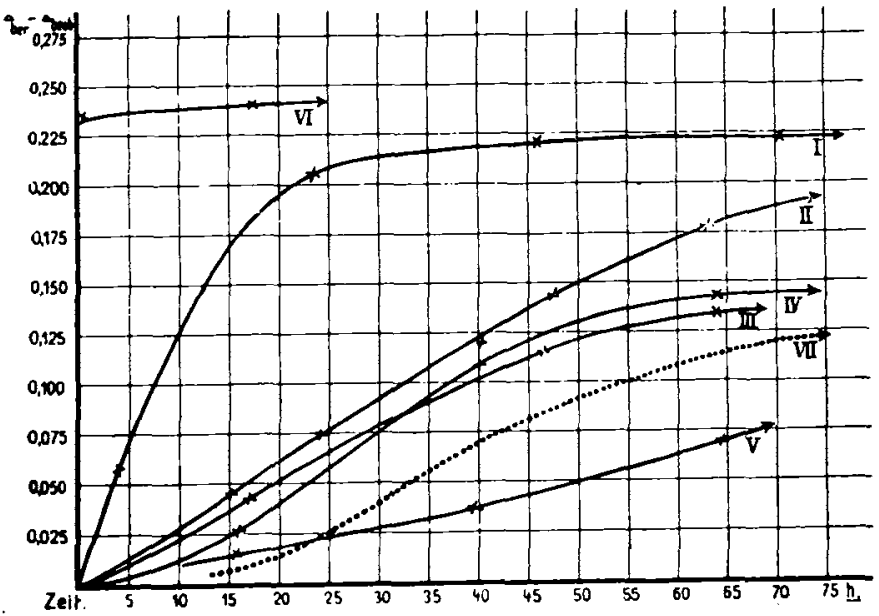

Figur 2.5)

I. Benzylhydroxylamin + dceton;

$\begin{array}{rllll}\text { II. } & , & \text { + Methyläthylketon; } & " & 1: 5 \\ \text { III. } & " & \text { + Methylbutylketon; } & " & 1: 4 \\ \text { IV. } & " & \text { + Methylhexylketon; } & " & 1: 5 \\ \text { V. } & " & \text { + Diäthylketon; } & " & 1: 5 \\ \text { VI. } & " & \text { + Methylbenzylketon; } & " & 1: 5 \\ \text { (VII. } & " & \text { + Aceton } & \end{array}$

Was zunächst auffällt, ist der Umstand, dass in der Figur 1 nur drei, in der Figur 2 hingegen sechs Kurven von den überhaupt möglichen sieben dargestellt sind. Es hat dies seinen einfachen Grund in der Reactionsträgheit der Ketone Methyläthyl-, Methylhexyl-, Diäthy]keton und Acetophenon. Denn die drei erstgenannten von ihnen besitzen erst bei höheren Concentrationen merkliche Finwirkung auf Benzylhydroxylamin. Und Acetophenon scheint sogar auch dann noch indifferent zu sein.

i) Figur 2 ist beziiglich der Werthe für $\Delta_{\text {ber. }}-\Delta_{\text {beob. }}$ in 21/2 mal kleinerem Maassstabe gezeichnet als Figar 1. Um aber mit letzterer einen Vergleich auch bei diesem reducirten Maassstabe zu ermüglichen, ist die Kurve I der Figur 1 hier nochmals eingezeichnet worden (Kurve TII). 
Iras die Reactionsgeschwindigkeit angeht, mit der sich die verschiedenen Ketone mit Benzylhydroxylamin zu vereinigen bestrebt sind, so erfährt sie durch die gezeichneten Kurven eine deutliche Illustration. indem ja die Depressionsabnahmen in Bezug auf die Zeit als ihr directes Maass anzusehen sind. Am reactionsfähigsten ist zweifellos das Methylbenzylketon, dem sich als nächstes das Aceton anschliesst. An dritter Stelle dürfte Methylbutylketon stehen, dem Methyläthyl- und Methylhexylketon als ungefähr gleichwerthig folgen. Diäthylketon ist schon relativ träge. Den grossen Einfluss der wirkenden Menge auf die Geschwindigkeit der Reaction lehrt ein Vergleich der Kurven II und VII der Figur 2 mit grosser Deutlichkeit.

Die beobachteten Depressionsabnahmen sind fast alle wohl der alleinigen Bildung von $\mathrm{N}$-Benzylketoximhydraten zuzuschreiben, da die Reactionsflüssigkeiten klar blieben. Lediglich beim Methylbenzylketon wurden 'Trübungen beobachtet, welche auf Fintstehung geringer Mengen von N-Benzylketoximäther hindeuten mögen. Als genügend sicherer Nachweis dieser Verbindungen konnte diese vereinzelte Beobachtung aber nicht angesehen werden.

Hatte somit die erste Versuchsreihe eigentlich nur den quantitativen Ausdruck für die qualitativ schon aufgefundene Thatsache der relativ leichten Bildung von N-Alkylketoximhydraten finden lassen, so erlaubte die zweite Versuchsreihe den ziemlich sicheren Nachweis der freien $\mathrm{N}$-Alkylketoxime selbst - ziemlich sicher, insofern die angewendete Methode ja nur zu Wahrscheinlichkeitsschlüssen, nicht aber zu einwandfreien Folgerungen führt.

B. Ueber das Verhalten von Ketonen gegenüber $\beta$-Benzylhydroxylamin bei Anwesenheit von Basen.

Die $z$ weite Versuchsreihe verfolgte den Zweck, die Trirkung von basischen Zusätzen auf die Reaction zwischen 
Ketonen und $\beta$-Benzylhydroxylamin kennen $z$ lernen, da ja die hierüber in der vorstehenden Mitheilung gemachten Angaben aus dem Grunde nicht ausreichend sein konnten, weil nur von solchen Versuchen definitive Fntscheidung zu erhoffen war, bei denen einmal gleiche Mengen von Keton und $\beta$-Benzylhydroxylamin obne basischen Zusat $\%$ und sodann $\mathrm{m}$ it einem solchen zur thwendning gelangten.

Als Ketone fanden die Mehrzahl der vorhin genannten Anwendung; betreff's der Basen beschränkte man sich auf Triäthylamin, Tribenzylamin und Dimethylanilin.

Vom $\beta$-Benzylhydroxylamin und den verschiedenen Ketonen wurden stets je 1 Millimol genommen, wohingegen die Mengen des Basenzusatzes auf 1 und 3 Millimole bemessen wurden. Die Ausführung der Bestimmungen geschah in durchaus der gleichen Weise wie vorhin. Als Lösungsmittel wurden deshalb wieder durchgängig je $15 \mathrm{~g}$ Benzol benutzt. Ebenso wurden die einzelnen Versuche auch wieder auf ca. $3 \times 24$ Stunden ausgedehnt.

Ueber die für nothwendig erachteten Vorversuche sei Folgendes mitgetheilt. Zunächst wurde das Verhalten der Basen nochmals in Benzol geprüft, damit auf Grund der dabei erhaltenen Depressionen die den anzuwendenden Concentrationen entsprechenden Constantenwerthe bei der Berechnung der einzelnen Versuchsdaten benutzt werden konnten. Erwähnt sei auch, dass bei diesen Versuchen insofern noch besonders vorsichtig verfahren wurde, indem Controlle der festgestellten Depressionen bis zur Zeitdauer von ca. 70 Stunden ausgeübt wurde. Eine genaue Untersuchung erstreckte sich sodann, wie auch schon früher, auf Feststellung der Stabilität der Systeme

sowie

$$
\text { Keton }+ \text { Base }
$$

Benzylhydroxylamin + Base. 
Der frühere Befund gegenseitiger Indifferenz der Componenten konnte nur bestätigt werden.

Der Einfluss nunmehr, den der Zusatz der drei genannten Basen auf die zwischen den Ketonen und Benzylhydroxylamin stattfindende Reaction ausübt, kann am besten aus den folgenden Schaubildern ersehen werden, welche die jeder Versuchsserie entsprechenden Depressionsdifferenzen-Zeit-Kurven wiedergeben.

Was zunächst den Reactionsverlauf zwischen Aceton und Benzylhydroxylamin angeht, so hat er in Figur 3 eine übersichtliche Darstellung gefunden.

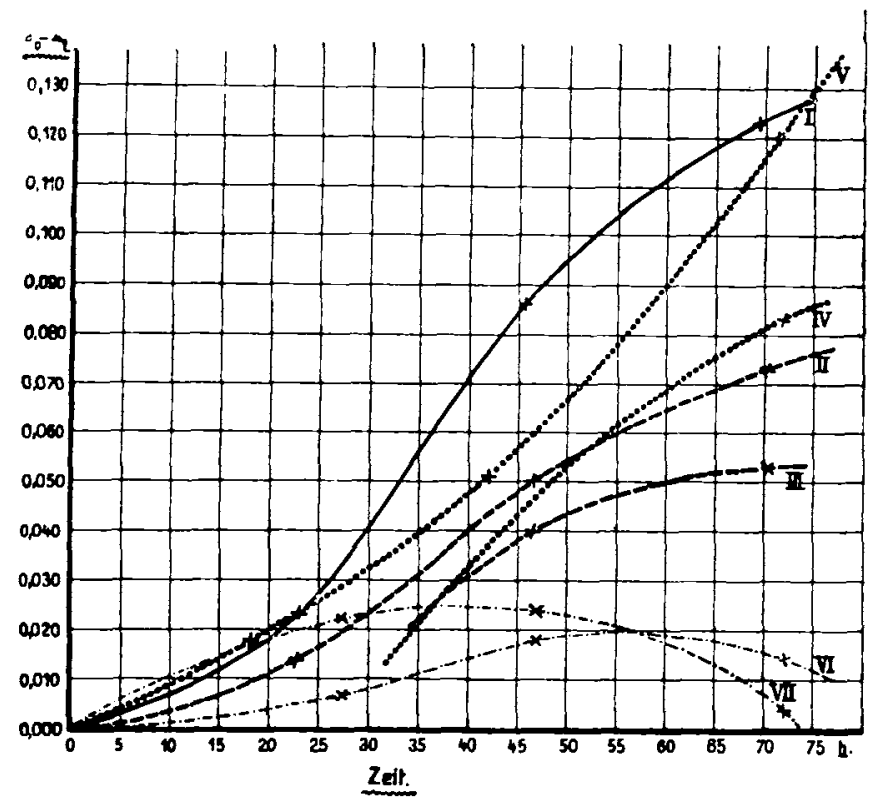

Figur 3.

I. Benzylhydr. + Aceton (ohne Base); Mol.-Verh.: $1: 1$

\begin{tabular}{|c|c|c|c|c|c|c|}
\hline \\
\hline \multirow[t]{2}{*}{11} & $"$ & + & $"$ & + Triäthylamin & $"$ & $1: 1: 1$ \\
\hline & $"$ & + & $"$ & + & $"$ & $1: 1: 3$ \\
\hline $\mathbf{1}$ & $"$ & + & $"$ & + Tribenzylamin & $"$ & $1: 1: 1$ \\
\hline & $"$ & + & " & $+\quad$, & $n$ & $1: 1: 3$ \\
\hline & " & t & " & + Dimethylanilin; &, & $1: 1: 1$ \\
\hline & $"$ & + & " & $+\quad$, & , & $1: 1: 3$ \\
\hline
\end{tabular}


Man gewinnt zunächst den Eindruck, als ob die Anwesenheit der Basen hemmend auf die Bildnng des $\mathrm{N}$-Benzylacetoximhydrats gewirkt habe. Hs erscheint indess hier nicht angängig, den Verlauf dieser Reaction nach dem Gange der Depressionsdifferenzen allein zu beurtheilen. Denn ausser dem zur Entstehung der Additionsverbindungen führenden Prozess

Keton +Benzylhydroxylamin $=$ N-Benzylketoximhydrat (1) kann sich ja auch noch ein zweiter Vorgang abspielen, welcher den eigentlichen $\mathrm{N}$-Aether selbst liefert

N-Benzylketoximhydrat $=\mathrm{N}$-Benzylketoxim + Wasser $(2$ ).

Es ist wohl als ziemlich sicher anzunehmen, dass neben der Reaction nach 1 auch die nach 2 in jedem Falle statthat. Die bisherigen Untersuchungen ohne Basen haben ja hiervon freilich nichts oder nur kaum etwas (Methylbenzylketon) spüren lassen, wie aus dem Klarbleiben der Reactionsmischungen, sowie aus dem einsinnigen Gang der Werthe für die Depressionsdifferenzen hervorging. Bei den unter Basenzusatz erhaltenen und vorstehend sowie im Folgenden graphisch wiedergegebenen Versuchsdaten ist nun aber zu berücksichtigen. dass z. Th. nicht nur Trübung der Reactionsmischungen - wenigstens kurz vor dem Gefrieren - eintrat, sondern dass die Depressionsdifferenzen z. Th, auch erst zunahmen und dann wieder abnahmen. Ein sich nach aussen hin durch diese Anzeichen dokumentirender Einfluss der basischen Substanzen ist somit unverkenubar, und es fragt sich nur noch, wie deren Einwirkung in jedem einzelnen Falle zu deuten ist. Beziiglich der Anzahl der in der Lösung befindlichen Moleküle - d. h. also des Factors, der die beobachteten Depressionen bestimmt - wirken nun die beiden in Betracht kommenden Reactionen ja einander entgegen. Die erste vermindert sie und bewirkt deshalb eine Vergrösserung der Depressionsdifferenzen, die zweite vermehrt sie, veranlasst also eine Verringernng dieser Werthe. Tritt nun ein die Anhydri- 
sirung begünstigendes Moment - hier ein Katalysator. basischer Natur - hinzu, so kann verschiedenerlei eintreten. Fs kann nämlich einmal die Verringerung und die Wiedervermehrung der Molekülzahl sich ungefähr das (ileichgewicht halten. Dieser Fall wird besonders dann statthaben, wenn das rerwendete Keton an sich nicht sonderlich reactionsfähig ist. Das gebildete Wasser kann alsdann in dem trockenen Benzol vollkommen, selbst beim Abkühlen, gelöst bleiben, und die Folge ist, dass die Reactionsfähigkeit des betreffenden Ketons durch den gemachten Zusatz keine scheinbare Hemmung, aber auch keine sonderliche Förderung erfährt. Das Methylbutylketon bietet ein Beispiel hierfür, wie ein Blick auf die Fig. 4 sofort lehrt.

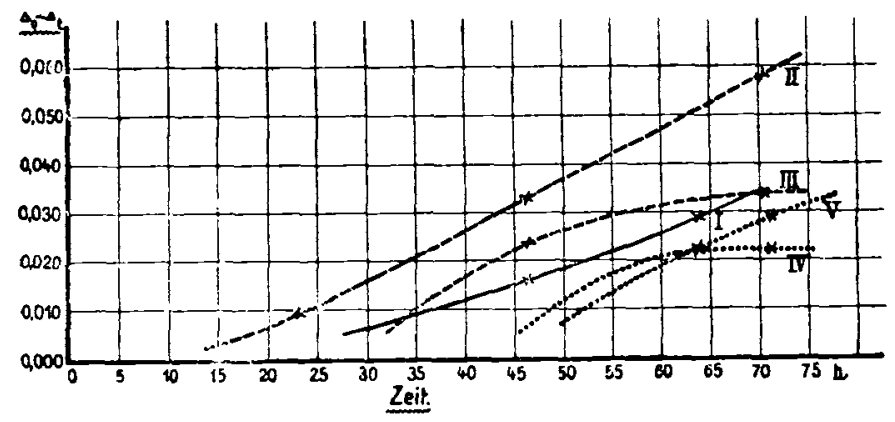

Figar 4.

I. Benzylhydr. + Methylbutylketon. (ohne Base); Mol.- Verh.: $1: 1$
II.
III.

Es kann die Anhydrisirung der wegen der Hydratbildung zunehmenden Depressionsdifferenz aber auch ziemlich stark entgegenwirken, sodass letztere in ihrem Gange in Folge Anwesenheit des Katalysators eine nicht unerhebliche Verzögerung erfährt. Dies wird sich besonders bei reactionsfähigen Ketonen, wie beim Aceton, zeigen und kann dann leicht als hemmender Finfluss des Katalysators gedeutet werden. Da aber in solchen 
Fällen die Menge des bei der Anhydrisirung entstehenden Wassers zu gross ist, um - wenigstens beim Abküblen gelöst zu bleiben, so bietet das Eintreten eine Trübung des benzolischen Reactionsgemisches vor dem Gefrieren einen deutlichen Hinweis auf die in Wahrheit stattfindenden Vorgïnge. Die Kurven II, III und IV der Figur 3 illustriren die eben erörterten Verhältnisse sehr deutlich.

Zum dritten endlich kann auch erst ziemlich weitgehende Hydratbildung statthaben, der dann in Folge Anwesenheit des Katalysator's eine stetig zunehmende Anhydrisirung folgt. Alsdann werden die Depressionsdifferenzen erst anwachsen und darauf wieder abnehmen, was also zu Kurven führt, wie sie in der folgenden Figur 5 für Nethylbenzylketon erhalten worden sind (Kurven II, IV u. V). Die Trübungen der Reactionsmischungen können hier erklärlicherweise besonders stark werden.

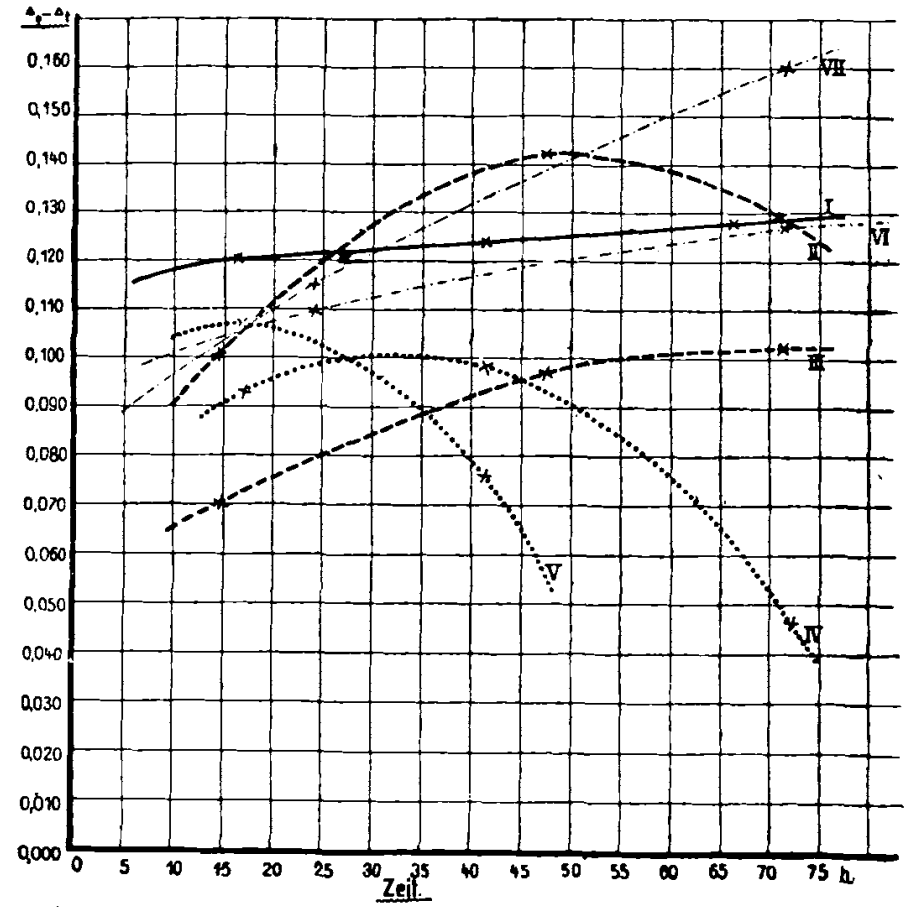

Figur 5. 


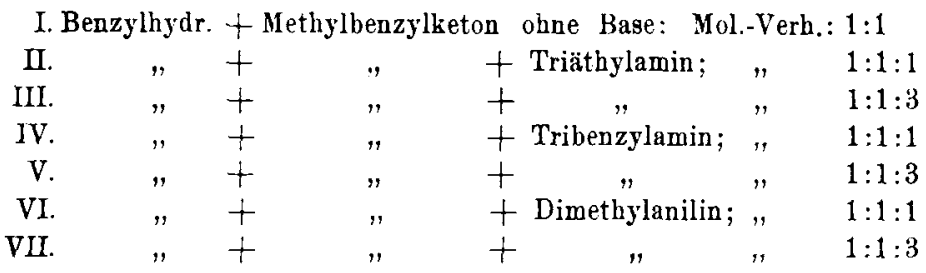

Es ist natürlich klar, dass sich auch alle möglichen Uebergänge $z$ wischen den drei als besonders charakteristisch heraus gegriffenen Fällen finden werden. Etwas Neues bieten die hierüber vorliegenden Kurvenbilder aber nicht, weshalb deren Wiedergabe unterbleiben kann.

Wenngleich zugegeben werden muss, dass hiermit der Nachweis der freien N-Benzylketoxime auch noch nicht den Grad der Sicherheit erlangt hat, welchen eine directe Isolirung dieser Substanzen für sich in Anspruch nehmen dürfte, so kann doch immerhin das nunmehr erzielte Ergebniss insofern als vorläufig befriedigend gelten, als es das unterschiedliche Verhalten der Aldehyde und Ketone gegegenüber $\beta$-substituirten Hydroxylaminen nicht als Unterschied der Art, sondern nur des Grades erkennen lehrt. Für weitere, auf die Isolirung der $\mathrm{N}$-Alkylketoxime selbst gerichtete Versuche ist damit wenigstens eine sicherere Grundlage als die bisherige geschaffen. Zunächst freilich wird es sich erst darum handeln, das Verhalten der Ketone gegenüber $\mathbf{N}$-Arylhydroxylaminen in analoger Weise zu studiren, wie es in diesen Mittheilungen für $\beta$-Benzylhydroxylamin beschrieben wurde. 